\title{
Drittwirkung von Schutzpflichten im Netz
}

\section{Einführung}

\section{Das Phänomen der Vertragsnetze}

Vertragsnetze können der äußeren Erscheinung nach in Vertragsketten in Erscheinung treten. ${ }^{1}$ So folgen im produzierenden Gewerbe auf Einkäufe (von Rohstoffen, Grundstoffen, Einbauteilen etc.) wieder die Verkäufe des daraus hergestellten Produkts. Weiter denke man an Lieferketten im Handel, bei der Energielieferung (z.B. Rohöl) oder an hintereinander geschaltete Verträge im Transportbereich. ${ }^{2}$ Aber auch die Abwicklung des bargeldlosen Zahlungsverkehrs, bei der Bank, Girozentrale, Clearingstelle und eine weitere Bank hintereinander geschaltet sind, ist ein Beispiel hierfür. Zudem ist an Vermietung und Weitervermietung bzw. Untermiete zu denken. ${ }^{3}$

Daneben sind Vertragsnetze in Gestalt sternförmiger Verbindungen zu beobachten. In diesem Fall schließt eine (natürliche oder juristische) Person mit vielen anderen Vertragspartnern ähnliche Verträge. Besonders augenfällig wird dies beim Arbeitgeber. Soweit es um Verträge mit selbstständigen Wirtschaftssubjekten geht, ist das Franchising das bekannteste Beispiel. Dabei kommt die Besonderheit hinzu, dass der einzelne Franchisenehmer wieder eine Vielzahl von Verträgen mit seinen Lieferanten oder Abnehmern schließt. So entstehen weitere sternförmige Unterverbindungen, so dass das gesamte Vertragsnetz an das Bild eines stilisierten Schneesterns erinnert.

Bei sternförmigen Verbindungen laufen die Vertragsbündel in der Zentrale zusammen. Die auf der anderen Seite stehenden Vertragspartner haben rechtlich unmittelbar nichts miteinander zu tun. Sie mögen sich mehr oder weniger gut kennen (wie z.B. Arbeitnehmer im selben Betrieb, Franchisenehmer in der gleichen Stadt), oder auch überhaupt keine Vorstellung von Art und Anzahl der anderen Vertragspartner haben. Je nachdem, ob und inwieweit auch eine Kooperation oder Abstimmung der einzelnen »Systempartner « untereinander erforderlich ist, nimmt man voneinander Kenntnis oder auch nicht. Auf der Einkaufsseite bilden sich ähnliche Vertragsnetze, wenn Hersteller komplexer Produkte, z.B. Automobilhersteller, mit zahlreichen Zulieferern zusammenarbeiten, die wiederum von Sublieferanten beliefert werden. Ähnliche, aber wieder etwas andersartige Netze bilden sich bei Projektverträgen, wie sie im Rahmen größerer Bau- oder Infrastrukturprojekte (z.B. Flughafen) zustande kommen. Hier wirken Projekterrichter, Projektbetreiber, Konzessionsgeber, finanzierende Banken und

1 Dazu Hugh Collins (2006) »The Weakest Link: Legal Aspects of the Network Architecture of Supply Chains «, in: Marc Amstutz und Gunther Teubner (Hg.) Contractual Networks: Legal Issues of Multilateral Cooperation, Oxford: Hart, im Erscheinen.

2 Vgl. Karl Larenz und Manfred Wolf (2004) Allgemeiner Teil des bürgerlichen Rechts, 9. Aufl., München: Beck, § 23 Rn. 128 f.

3 Dazu auch Marc Amstutz (2004) »Die Verfassung von Vertragsverbindungen«, in diesem Heft S. 105 ff. 
eine Vielzahl von Generalunternehmern und Subunternehmern zusammen, die durch stern- und kettenförmige Verträge miteinander verbunden sind. Es entstehen Unternehmensnetzwerke.

Das Phänomen der Vertragsnetze ${ }^{4}$ wirft für das deutsche Recht die Frage auf, ob unser überkommenes Schuldrecht, das den Kategorien von Vertrag und Gesellschaft verhaftet ist, noch ausreicht, um die mit Vertragsnetzen verbundenen Rechtsfragen zu lösen; oder ob es notwendig ist, sich um eine entsprechende Rechtsfortbildung zu bemühen. Denn man ist sich überwiegend einig darüber, dass diese Vertragsnetze zumindest der äußeren Erscheinung nach eine neue Kategorie zwischen Markt und Hierarchie, zwischen Vertrag und Verband bilden. ${ }^{5}$ Es kommt zwar wie bei Gesellschaften $\mathrm{zu}$ einer Kollision eigennütziger und gemeinnütziger Interessen, doch mit dem Unterschied, dass das Verhältnis von Eigen- und Fremdnützigkeit im Netz reziprok ist. Vertragsnetze nehmen insofern eine Zwischenstellung zwischen Interessenverbindung, Interessenwahrung und Interessengegensatz ein. Die Interessenstruktur ist gekennzeichnet durch ein Spannungsverhältnis von Systembindung einerseits und Autonomie, Eigenzweckverfolgung ${ }^{6}$ und Rivalität andererseits. ${ }^{7}$ Die Individualorientierung bleibt zwar im Vordergrund, die Kollektivorientierung wird aber je nach Fall mehr oder weniger deutlich erkennbar und prägend. ${ }^{8}$ Schließlich ist zur Erreichung des jeweiligen übergeordneten Ziels eine Abstimmung aufeinander und eine Form von Kooperation notwendig.

Demgemäß spricht man vom Verbundzweck, Netzzweck oder Systemzweck. ${ }^{9}$ Dieser mag in der Herstellung eines erfolgreichen Endprodukts, in der Durchführung eines Großprojekts oder in der erfolgreichen Vermarktung eines Produkts liegen. Bei den einzelnen Verträgen geht es zwar - wie bei sonstigen Verträgen auch - primär um die störungsfreie Durchführung des jeweils vereinbarten Leistungsaustausches. Über diesen allgemeinen Sinn und Zweck von Verträgen hinausgehend dient der einzelne Vertrag im Netz mittelbar jedoch auch der Erreichung des alle Mitglieder verbindenden Netzzwecks. Allerdings erreicht dieser Zweck auch wieder nicht die Qualität eines Zwecks im engeren Sinne des Gesellschaftsrechts (§ 705). Denn dort ist der Zweck primärer Vertragsgegenstand. Es geht vielmehr um die dazwischen liegende Kategorie, die - bei aller denkbaren Kritik am Begriff »Zweck« - doch am griffigsten mit dem Ausdruck »Netzzweck « zu erfassen ist.

4 Gegen den Begriff »Netz« hat sich allerdings Peter Krebs (2000) Sonderverbindung und außerdeliktische Schutzpflichten, München: Beck, 314 f., ausgesprochen; schließlich ginge es immer nur um Ketten von Verträgen, nicht wirklich um Netze. Dagegen ist allerdings einzuwenden, dass der Begriff - ähnlich wie das Schlagwort vom »Netzwerk« - ohnehin nicht streng bildlich gemeint ist.

5 Vgl. Mathias Rohe (1998) Netzverträge, Tübingen: Mohr Siebeck, 491.

6 Reinhard Böhner (1998) »Werbekostenzuschüsse und sonstige Einkaufsvorteile in Franchisesystemen «, 51 NJW, 109-112.

7 Meines Erachtens geht es jedoch zu weit, von widersprüchlichen Verhaltensanforderungen zu sprechen, so aber Gunther Teubner (2004) Netzwerk als Vertragsverbund, Baden-Baden: Nomos, 80.

8 Gunther Teubner (2004) »Profit sharing als Verbundpflicht?«, 168 ZHR, 78-96, 86.

9 Teubner (Fn. 8) 85 m.w.N. 


\section{Neuer rechtlicher Handlungsbedarf?}

Doch resultiert aus der beschriebenen, besonderen Interessenstruktur wirklich neuer rechtswissenschaftlicher Handlungsbedarf? Das ist nur dann der Fall, wenn diese Vertragsnetze neuartige Rechtsfragen auslösen, die mit Hilfe des geltenden Rechts und seiner Instrumente, insbesondere der Vertragsauslegung, nicht angemessen zu lösen sind. Manche bejahen dies und fordern die Anerkennung neuer Rechtsinstitute. ${ }^{10}$ Das gilt etwa für Möschel ${ }^{11}$ und Rohe ${ }^{12}$, die sich für den willensunabhängig entstehenden sog. »Netzvertrag « zwischen den Netzmitgliedern ausgesprochen haben. Aufgrund der massiven dogmatischen Bedenken konnte sich dieser Vorschlag jedoch nicht durchsetzen. ${ }^{13}$ Schließlich räumt Rohe selbst ein, dass sich viele der gewünschten Ergebnisse schon allein im Wege der Vertragsauslegung erreichen lassen. Und insofern mag sein Hinweis auf den Netzzweck durchaus seine Berechtigung haben; abzulehnen ist jedoch die daran anknüpfende, weitergehende Forderung nach einem Netzvertrag.

Tatsächlich fragt sich auch, wo die Probleme liegen, die nicht schon durch eine Regelung im einzelnen Vertrag bewältigt werden können; zumal innerhalb des Netzes auch viel durch die Vereinheitlichung der Verträge, insbesondere durch die Verwendung einheitlicher allgemeiner Geschäftsbedingungen, oder auch durch Bezugnahme auf andere Verträge erreicht werden kann. Unter Umständen kann auch - freilich im Rahmen der $\S \S 305$ ff. BGB - vorgeschrieben werden, welche Inhalte Subunternehmerverträge etc. haben sollen. ${ }^{14}$ Weiterhin besteht auch zwischen Parteien im Netz, die selbst nicht durch Vertrag miteinander verbunden sind, kein rechtsfreier Raum. Bei Verletzung konkreter Rechtsgüter bietet das Deliktsrecht, §§ 823 ff. BGB, hinreichenden Schutz. In krassen Fällen deckt § 826 BGB auch reine Vermögensbeschädigungen ab. ${ }^{15}$ Zudem darf das vertragsunabhängig wirkende Produkthaftungsgesetz nicht vergessen werden.

$10 \mathrm{Zu}$ erwähnen sei auch die These vom trilateralen Synallagma, die Heermann entwickelt hat, vgl. Peter Heermann (1998) Drittfinanzierte Erwerbsgeschäfte, Tübingen: Mohr Siebeck. Diese wurde aber für drittfinanzierte Erwerbsgeschäfte (vgl. a.a.O. 306) und nicht für Verträge im Netz entwickelt, weshalb sie hier von geringerer Bedeutung ist.

11 Wernhard Möschel (1986) »Dogmatische Strukturen des bargeldlosen Zahlungsverkehrs«, 186 AcP, 185-236, 211.

12 Rohe (Fn. 5); im Mittelpunkt seiner Betrachtungen steht der bargeldlose Zahlungsverkehr. Rohe will seine Thesen aber auch auf andere Netze erstrecken, sofern ein besonderer innerer Zusammenhalt durch Sekundärtransaktionen gegeben ist, also z.B. bei Franchising, Vertragshändlernetzen oder auch bei der Just-in-Time-Produktion, a.a.O. 498.

13 Siehe z.B. Kritik bei: Uwe Hüffer (1987)»Die Haftung gegenüber dem ersten Auftraggeber im mehrgliedrigen Zahlungsverkehr«,151 ZHR, 93-117, 106 f.; Helmut Heinrichs (2005) vor $\S 305$, in: Palandt: Bürgerliches Gesetzbuch, 64. Aufl., München: Beck, vor $\S 305$ Rn. 16; Karsten Schmidt (1999) Handelsrecht, 5. Aufl., Köln: Heymanns, § 35 III; Eberhard Schwark (1987) »100 Bände BGHZ - Bank-und Wertpapierrecht«, 151 ZHR, 325354, 339. Weitere Nachweise zur Kritik bei Markus Stoffels (2001) Gesetzlich nicht geregelte Schuldverträge, 268 Fn. 442 und Teubner (Fn. 7) 104, Fn. 114.

14 Vgl. Fritz Nicklisch (2000)»Vernetzte Projektverträge und vernetzte Streitbeilegungsverfahren «, $55 B B, 2166-2169$.

15 S. dazu auch unten IV.2.f). 
Daher fragt sich, wo die Fälle sind, die zwangsläufig nach neuen rechtlichen Konstruktionen, nach Schutzbereichserweiterungen oder der Kreation neuer Sonderverbindungstatbestände rufen. ${ }^{16}$ Gibt es so viele einschlägige Fallkonstellationen mit messbaren Schäden und klagewilligen Parteien, dass sie die Entwicklung eigenständiger Rechtssätze rechtfertigen? Führt das Stehenbleiben bei den anerkannten Rechtsinstituten wirklich zu Gerechtigkeitsdefiziten, so dass eine innovative Lösung unumgänglich wird?

Im Grundsatz wird hier die These vertreten, dass das geltende Recht im Hinblick auf die Situation in Vertragsverbünden seiner Ordnungsaufgabe noch sehr weitgehend gerecht wird. Das heißt aber nicht, dass das Recht das Phänomen von Vertragsnetzen ${ }^{17}$ ignorieren sollte. Im Gegenteil: Die Aufgabe heißt vielmehr, netzspezifische Besonderheiten zu erkennen, um sie bei der Vertragsauslegung, Pflichtenbestimmung und AGB-Kontrolle ${ }^{18}$ angemessen berücksichtigen zu können, soweit dies notwendig erscheint, um die gegebenen Interessenstrukturen hinreichend erfassen zu können. Demgemäß soll im Folgenden untersucht werden, ob und inwieweit sich die Netzeinbindung des einzelnen Vertrags auf dessen Pflichtenprogramm auswirkt. ${ }^{19}$

\section{Die Netzeinbindung des Vertrags als pflichtenbestimmendes Kriterium}

\section{Der einzelne Vertrag als Ausgangspunkt der Betrachtung}

Mit Teubner ist dabei als Ausgangspunkt und Mittelpunkt der Betrachtung der einzelne Vertrag und seine differenzierte Interessenstruktur im Einzelfall zu wählen; zumal im Netz die Individualorientierung ausschlaggebend ist. ${ }^{20}$ Der Wille des Einzelnen ist primär auf den Abschluss eines konkreten Vertrags gerichtet und nur sekundär auf die Verfolgung des übergeordneten Netzzwecks. Regelmäßig kennen die einzelnen Vertragspartner auch nur den Inhalt des eigenen Vertrags und nicht die sonstigen Verträge im Netz. Insofern ist klar, dass die jeweiligen Einzelinteressen dominieren. Jeder will für sich soviel Profit wie möglich herausholen. Solange dies auch mit Profit

16 Denn siehe Motive II, S. 2: Außer Zweifel steht, dass durch das Schuldverhältnis nur persönliche Rechtsbeziehungen zwischen den in demselben Vertrag stehenden Personen begründet werden, der persönliche Anspruch des Gläubigers gegen den Schuldner auf die Leistung, die persönliche Verbindlichkeit des Schuldners zur Bewerkung der Leistung (...). Über die Personen des Gläubigers und Schuldners greift die Wirkung des Schuldverhältnisses an sich nicht hinaus.

17 Wenig tauglich für die juristischen Betrachtungen erscheint aber der eher sozialwissenschaftliche Begriff vom »Netzwerk«; zu den Begriffen auch Jean Druey (2006) »Das Recht als Netz für Netzwerke: Eine Wegskizze«, in diesem Heft I.4.

18 Dies wird allerdings in der Rechtsprechung auch schon erkennbar, vgl. BGHZ 77, 126, 133; BGH NJW 1981, 117, 119; 1982, 178, 180; BGH WM 1984, 986; siehe auch Rohe (Fn. 5) 296.

19 Dazu schon Teubner (Fn. 7) $156 \mathrm{ff}$.

20 Gunther Teubner (2004) »Coincidentia oppositorum: Das Recht der Netzwerke jenseits von Vertrag und Organisation «, in: Marc Amstutz (Hg.) Die vernetzte Wirtschaft: Netzwerke als Rechtsproblem, Zürich: Schulthess, 7-38, 11, 23. 
für den Vertragspartner verbunden ist, ist das gut, aber letztlich zweitrangig. Außerdem ist jeder für sich bemüht, eigene Risiken soweit wie möglich zu minimieren bzw. Risiken aller Art auf andere zu verlagern. ${ }^{21}$ Der Schauplatz des Geschehens und der rechtliche Anknüpfungspunkt bleibt daher zunächst der einzelne Vertrag.

\section{Die Bedeutung der Einbindung des Vertrags ins Netz.}

Indessen sind sich alle, die sich mit dem Netzphänomen auseinander setzen, einig darüber, dass die Eingebundenheit des Vertrags in das Netz bzw. den Vertragsverbund Auswirkungen auf die Vertragsverhältnisse hat, die zu berücksichtigen sind. ${ }^{22}$ Und in der Tat weist die Einbettung des einzelnen Vertrags in das Netz Besonderheiten auf, die über die im Wirtschaftsleben fast überall zu beobachtende, übliche Verknüpfung von Verträgen in bezeichnender Weise hinausgeht. So ist für den Netzverbund kennzeichnend, dass dessen Grenzen hinreichend bestimmt oder doch zumindest für alle Mitglieder bestimmbar sind, eben z.B. die Zahl und der Sitz der Systempartner eines Franchisesystems oder der Just-in-time-Zulieferer eines Herstellers komplexer Produkte. Weiterhin verbindet alle auf der sekundären Interessenschiene der übergeordnete, gemeinsam verfolgte Zweck. Dieser Netzzweck wird mehr oder weniger ausdrücklich zum Inhalt aller Verträge gemacht. Demgemäß kommt es auch oft zu einem einheitlichen Auftreten nach außen. In der Gesamtsicht lässt sich von systematischen, auf Dauer angelegten und auf gegenseitigem Vertrauen ${ }^{23}$ aufbauenden Kooperationsgemeinschaften sprechen.

Für die Auslegung des einzelnen Vertrags und seines Pflichtenprogramms ist insoweit beachtlich, dass jeder Vertrag auch den übergeordneten Sinnzusammenhang in sich aufnimmt, der dann einzelfallorientiert aufzudecken und zu bewerten ist. ${ }^{24}$ Dem übergeordneten Netzzweck kann im einen Fall eine geringe, im anderen Fall aber auch eine sehr große Bedeutung zukommen, sodass er neue weitergehende Rechte und Pflichten begründen mag. Oft mögen dabei netzbezogene Rechte, Pflichten und Zielbestimmungen schon von den Parteien selbst ausdrücklich zum Vertragsinhalt gemacht worden sein. Aber auch dann ergibt sich zumindest im Rahmen der AGB-Kontrolle das Problem, die Grenzen etwaiger Rechte und Pflichten zu bestimmen und entsprechende Bewertungsmaßstäbe zu entwickeln. Fehlen konkrete Abmachungen, fragt sich weiter, welche Rechte und Pflichten sich allgemein aus der Netzeinbindung des einzelnen Vertrags ableiten lassen. Es geht also um die Bestimmung des Inhalts und der Reichweite von netzspezifischen Neben- bzw. Schutzpflichten. Verbunden damit stellt sich die Frage, ob etwaige Schutzpflichten nur zwischen den Vertragsparteien oder ob sie auch zwischen nicht vertragsmäßig miteinander verbundenen Netzmitgliedern Wirkung entfalten können.

21 Vgl. dazu beim Projektvertrag auch Nicklisch (Fn. 14) 2168.

22 Knut Lange (1998) Das Recht der Netzwerke, Rn. 465; Möschel (Fn. 11) 211 und 235.

23 Vgl. Teubner (Fn. 7) 42.

24 Stoffels (Fn. 13) 262. 
III. Die Inhaltsbestimmung der Schutzpflichten von Netzmitgliedern

\section{Kooperationspflichten}

a) Allgemeines

Netzverbünde sind dadurch gekennzeichnet, dass eine Vielzahl von Parteien in organisierter, systematischer Weise zusammenwirkt. Insofern sind sich auch alle darüber bewusst, dass ein gewisser Grad an Kooperation erforderlich ist, damit das System funktioniert. ${ }^{25}$ Und das ist wieder Voraussetzung dafür, dass der einzelne Vertrag seinen Zweck erfüllen kann. Mag auch der eigene Profit an erster Stelle stehen, so ist das Interesse aller doch zugleich auf das erfolgreiche Zusammenwirken gerichtet, damit ein wettbewerbsfähiges Produkt entsteht, bargeldlose Zahlungsströme abgewickelt werden können etc. Denn von diesem »Enderfolg«, von der fortwährenden, komplikationslosen Erreichung des Netzzwecks ist letztlich auch die Existenz der einzelnen Netzmitglieder abhängig.

Aus dieser Kooperationsnotwendigkeit heraus wird man recht zwanglos auf Kooperationspflichten schließen können. Sie können etwa auf die Weitergabe erhaltener Informationen bezogen sein oder auf die Dokumentation bestimmter Vorgänge. Die Netzpartner mögen auch allgemein gehalten sein, stets die Folgen ihrer Handlungen für das gesamte Netz mit in die Betrachtung einzubeziehen und die Wechselwirkung der einzelnen Vertragsbeziehungen miteinander zu beachten. ${ }^{26}$ Allerdings ergibt sich eine Kooperationspflicht in diesem Sinne schon aus den jeweiligen einzelnen Verträgen und ihren Leistungszielen. Schließlich finden ein unmittelbarer Kontakt und ein direkter Leistungsaustausch auch nur zwischen den jeweils vertragsmäßig miteinander verbundenen Parteien statt. Eine aktive Pflicht zu bestimmtem Zusammenwirken wird sich zwischen Nicht-Vertragspartnern auch weder beim Franchising noch bei Zulieferketten etc. feststellen lassen. Hierfür besteht auch keine Notwendigkeit. Ist im Einzelfall etwas anderes gewollt, werden ohnehin konkrete Vereinbarungen getroffen werden, z.B. über Forschungskooperationen oder gemeinsame Werbeaktionen.

Als Fazit lässt sich daher festhalten, dass Kooperationspflichten regelmäßig nicht über das einzelne Vertragsverhältnis hinausgreifen. Zwar mag der Netzzweck Art und Umfang der Kooperationspflichten zwischen den Vertragspartnern mitprägen, ein unmittelbarer Drittbezug ist jedoch nicht erkennbar.

\section{b) Loyalitätspflichten}

Teilweise wird behauptet, dass die Mitglieder des Netzes Loyalitätspflichten gegenüber dem Gesamtnetz träfen. ${ }^{27}$ Das bleibt in dieser Form jedoch vage. Das gilt auch für den Hinweis darauf, dass Netzmitglieder Aktionen zu unterlassen hätten, die das Erreichen des Vertragszwecks gefährden. ${ }^{28}$ Insofern greift der Pflichteninhalt wohl auch noch nicht über die zuvor geschilderte allgemeine Kooperationspflicht hinaus.

25 Vgl. auch Herbert Wiedemann und Oliver Schultz (1999) »Grenzen der Bindung bei langfristigen Kooperationen«, 20 ZIP , 1-12, 3, die von »Kooperationsgemeinschaft« sprechen.

26 Lange (Fn. 22) Rn. 1449.

27 Teubner (Fn. 8) 89.

28 Lange (Fn. 22) Rn. 430. 
Interessant ist jedoch die Frage, ob die Kooperations- oder Loyalitätspflicht einer Kündigung zur Unzeit Grenzen setzt. Den $\S \S 627$ II, 671 II, 675 Hs. 2 und 723 II BGB lässt sich der allgemeine Rechtsgedanke entnehmen, dass eine Kündigung zur Unzeit zwar wirksam, aber rechtswidrig ist ${ }^{29}$ und daher ggf. zu einem Schadensersatzanspruch führen kann. ${ }^{30}$ Mit »Unzeit « ist dabei gemeint, dass der Kündigende zwar zur Kündigung berechtigt ist, hierzu aber ohne gerechtfertigten Grund ${ }^{31}$ einen Zeitpunkt wählt, der die Interessen der anderen Seite, z.B. bei der Gesellschaft die gemeinschaftlichen Interessen der Mitgesellschafter, verletzt. ${ }^{32}$ Der Anwendungsbereich dieses Grundsatzes ist jedoch eng. Erfasst werden nur solche Kündigungen, die an keine Frist gebunden sind und die den anderen Teil deshalb hart treffen, weil ihm nicht genügend Zeit zur Umstellung gelassen wird. ${ }^{33}$ Der Schutzzweck dieses Kündigungsverbots bezieht sich also darauf, dem Kündigungsempfänger eine angemessene Frist für die erforderliche Umdisponierung zu sichern. ${ }^{34}$

Auf das Netz bezogen könnte man sich - etwa bei Bauprojekten oder bei Just-inTime-Lieferungen - die Situation vorstellen, dass ein Partner zwar seine vertraglichen Kündigungsfristen einhält, der gewählte Zeitpunkt jedoch mit den Interessen anderer Netzmitglieder nicht in Einklang zu bringen ist und bei diesen dann, z.B. wegen Projektverzögerung, Schäden auslöst. Dann geht es zwar nicht um die Verletzung vertraglicher Pflichten, vielleicht aber um die Verletzung einer Verbundtreupflicht. Ein weiteres Beispiel bietet ein vom BGH entschiedener Franchising-Fall. Dort hatte der Franchisingnehmer, der zur Unterhaltung eines Transport- und Distributionssystems für im Kühlverkehr zu befördernde Lebensmittel verpflichtet war, fristlos gekündigt und damit für ein bestimmtes Gebiet das Verteilersystem lahm gelegt. Somit bestand die Gefahr, dass die Kühlkostware nicht rechtzeitig weiter transportiert wurde und verdarb. ${ }^{35}$ Auch hier könnte man überlegen, ob der Ausübung eines etwaig gegebenen Kündigungsrechts eine Verbundtreupflicht zeitweilig entgegenstehen oder statt zur

29 Vgl. Claus-Wilhelm Canaris (1979) »Kreditkündigung und Kreditverweigerung gegenüber sanierungsbedürftigen Bankkunden«, 143 ZHR, 113-138, 114; Klaus Hopt und Peter Mülbert (1998) Kreditrecht, Bankkredit und Darlehen im deutschen Recht, Kommentar, Berlin: Schweitzer, § 609 Rn. 23, 32; für Unwirksamkeit OLG Hamm NJW-RR 1986, 208, 209.

30 Peter Ulmer (2004) » 723«, in: Franz Säcker und Kurt Rebmann (Hg.) Münchener Kommentar zum Bürgerlichen Gesetzbuch, 4. Aufl., München: Beck, Rn. 55; Canaris (Fn. 29) 115; zu ersetzen sind allein die Nachteile, die dem anderen Teil durch die treuwidrige Wahl des Kündigungszeitpunkts entstanden sind, vgl. Ulmer a.a.O. § 723 Rn. 56.

31 Zulässig ist eine Kündigung zur Unzeit, wenn ein wichtiger Grund hierfür vorliegt, vgl. Ulmer (Fn. 30) § 723 Rn. 54; dieser Grund muss sich allerdings auch konkret auf den gewählten Zeitpunkt beziehen, a.a.O. Rn. 54.

32 Ulmer (Fn. 30) § 723 Rn. 53.

33 Z.B. soll einem Kreditnehmer, dem von der Bank gekündigt wird, eine Möglichkeit gegeben werden, umzuschulden bzw. die erforderliche Kapitalsumme zu besorgen, vgl. BGH WM 1978, 234; Canaris (Fn. 29) 113, 115; Klaus Hopt (1979) »Rechtspflichten der Kreditinstitute zur Kreditversorgung, Kreditbelassung und Sanierung von Unternehmen«, 143 ZHR, 139-173, 164.

34 Canaris (Fn. 29) 115.

35 BGH NJW 1999, 1177. 
fristlosen zur befristeten Kündigung verpflichten kann. Teubner scheint eine solche Verbundtreupflicht annehmen zu wollen. ${ }^{36}$ Darauf wird zurück zu kommen sein.

c) Systemförderpflicht beim Franchising

Im Bereich des Franchising wird speziell auch von einer Systemförderpflicht gesprochen. Gemeint ist damit u.a. eine Pflicht der Systempartner zu gleichförmigem Verhalten. Das betrifft die Gestaltung der Ladenlokale, der Verpackung, der Herstellungsmethoden, Werbung etc. Schließlich ist die Funktionsfähigkeit des Systems in hohem Maße von einem homogenen Erscheinungsbild des Produkts und einer Standardisierung der Qualität abhängig. ${ }^{37}$ Der Kunde soll das Produkt sofort wieder erkennen und überall gleich hohe Qualitätsansprüche stellen können. Auf diese Weise wird das Vertrauen des Kunden in dieses Produkt gestärkt, wovon wiederum alle Systempartner profitieren. Voraussetzung ist aber eben, dass sich alle strikt an die jeweiligen Vorgaben und Qualitätsstandards halten.

Berühmt geworden ist der vom BGH entschiedene Grilltemperatur-Fall, der die Kündigung eines McDonald's-Partners betraf, der nicht die exakt vorgeschriebene Grilltemperatur für Hamburger eingehalten hatte. ${ }^{38}$ Bei einer solchen, eher geringfügig wirkenden Pflichtverletzung mag die Kündigung auf den ersten Blick unverhältnismäBig erscheinen; berücksichtigt man jedoch die zuvor genannten Aspekte, die eine allgemeine Systemförderpflicht zum Schutz der Marke selbst wie auch unter Präventionsgesichtspunkten fordern, ist die Kündigung als gerechtfertigt anzusehen. Insoweit lässt sich zwar auch keine unmittelbare Drittwirkung von Vertragspflichten feststellen. Jedoch zeigt sich hier sehr deutlich, dass eine Regelung, die im Einzelvertragsverhältnis vielleicht als unverhältnismäßig erscheinen mag, aufgrund der Netzeingebundenheit einem anderen Bewertungsmaßstab unterliegt. Hieraus ergeben sich dann in der Tat gesteigerte Pflichten des Einzelnen.

Korrespondierend damit wird man eine Pflicht der Systemzentrale zur Überwachung der Systempartner zu bejahen haben. Insoweit liegt es im Interesse aller, dass die Wahrung der vorgegebenen Standards durch alle Netzmitglieder von der Systemzentrale auch kontrolliert wird und dass im Ernstfall rechtzeitig gegen etwaige »Freerider« eingeschritten wird, damit größerer Schaden für das Gesamtsystem und das Produktimage vermieden werden kann.

\section{Informationspflichten}

\section{a) Information über den eigenen Wirkungskreis}

Eine Konsequenz aus dem Netzzusammenhang können weiterhin spezifische Informations-, Hinweis- oder Warnpflichten sein. ${ }^{39}$ Das fängt bei vorvertraglichen Informations- und Offenbarungspflichten an. So wäre der potentielle neue Systempartner gehal-

36 Siehe auch Teubner (Fn. 8) 90.

37 Vgl. BGH NJW 1985, 1894, 1895.

38 BGH NJW 1985, 1894.

39 Dazu auch Teubner (Fn. 7) $159 \mathrm{f}$. 
ten, seine mangelnden Fähigkeiten oder Möglichkeiten, im Netz vereinbarungsgemäß mitzuwirken, offen zu legen. Unproblematisch erscheinen weiter Informationspflichten der Netzmitglieder über eigene Handlungen oder Unterlassungen. Bei Dauerschuldverhältnissen ergibt sich hier schon aus der allgemeinen Treupflicht, dass der andere Vertragspartner rechtzeitig über alle relevanten Veränderungen, über drohende Leistungsstörungen und Abwicklungsprobleme zu informieren ist. ${ }^{40}$ Das reicht vom Hinweis auf eine Materialänderung bis zur Ankündigung von verspäteten Lieferungen. In gleicher Weise sind die Systemzentrale, der Franchisegeber oder der Endprodukthersteller verpflichtet, auf geplante Änderungen im Netz, in der Vertriebsstruktur, im Belieferungssystem etc., rechtzeitig hinzuweisen. ${ }^{41}$ Alle Mitglieder sind auf demselben notwendigen Informationsstand zu halten. ${ }^{42}$ Allerdings richten sich diese Informationspflichten auch nur gegen die eigenen Vertragspartner. Gegen weitergehende Informationspflichten gegenüber Dritten spricht, dass man sich regelmäßig darauf verlassen darf, dass der eigene Vertragspartner die Information sogleich an seine Vertragspartner weitergibt, soweit diese auch davon betroffen sind. Insoweit darf man eben auch unterstellen, dass der Informationsfluss im Netz funktioniert.

Ist der einzelne Vertrag in ein Vertragsnetz eingebunden, kann die einzelne Leistungsstörung zu einer Art Kettenreaktion führen, wenn sich das Problem (z.B. eine Verspätung bei zeitgenau vereinbarten Lieferungen) auf den weiteren Ebenen fortsetzt. Der drohende Schaden kann sich dann vervielfachen. Die Hinweispflicht wird umso dringlicher. ${ }^{43}$ Allerdings ist das kein netzspezifisches Phänomen. Überall, wo Verträge hintereinander geschaltet sind, kann es auf diese Weise zu einer Potenzierung von Schäden kommen. Und insofern dürfte es auch allgemeiner Auffassung entsprechen, dass Hinweis- und Warnpflichten gegenüber dem Vertragspartner umso eher anzunehmen sind, je größer das jeweilige Schadensrisiko ist. Das gilt auch für den Fall, dass sich der Schaden erst auf einer späteren Ebene auswirkt; denn die Möglichkeit, dass dies doch noch mittelbar zur Inanspruchnahme des eigenen Vertragspartners durch Dritte oder zu sonstigen Nachteilen für ihn führt, ist wohl selten auszuschließen. Ein Grund für vertragsübergreifende Informationspflichten ist daraus aber bislang nicht hergeleitet worden.

40 Zur gesteigerten Bedeutung der Treupflicht bei Dauerschuldverhältnissen Dieter Medicus (2005) Schuldrecht I, München: Beck, Rn. 14; Hans-Leo Weyers (1982) »Typendifferenzierung im Werkvertragsrecht«,182 AcP, 60-79, 67; Peter Ulmer (1975) »Kündigungsschranken im Handels- und Gesellschaftsrecht - Zum Einfluss der Treupflicht auf die einseitige Vertragsbeendigung«, in: Wolfgang Hefermehl (Hg.) Festschrift für Philipp Möhring, München: Beck, 295-317, 296; Andreas Saxinger (1993) Zulieferverträge im deutschen Recht, Baden-Baden: Nomos, 147; Hartmut Oetker (1994) Das Dauerschuldverhältnis und seine Beendigung, Tübingen: Mohr Siebeck, 27.

41 Vgl. nur Rohe (Fn. 5) 439.

42 Teubner (Fn. 7) 160.

43 Siehe auch Rohe (Fn. 5) 404. 
b) Netzbezogene Hinweis- und Warnpflichten

Doch wie liegt es, wenn nicht Ursachen aus der eigenen Sphäre, sondern sonstige Umstände den Eintritt eines Schadens vermuten lassen? So könnte derjenige, der zwar nicht im eigenen Vertragsverhältnis, wohl aber an anderer Stelle im Netz einen drohenden Schaden ahnt oder Probleme kommen sieht, die Pflicht haben, diese Stelle darüber zu informieren, um weiteren Schaden zu vermeiden. Man denke vielleicht an die Warnung anderer Netzmitglieder vor der Schlechtleistung oder der Insolvenz ihrer Schuldner, die selbst wieder Netzmitglieder oder auch Dritte sein können. Es fragt sich jedoch, was mit der Annahme einer solchen Pflicht gewonnen wäre. Meist werden entsprechende Hinweise freiwillig erfolgen. Die Annahme einer Pflicht hätte also nur Bedeutung für den Fall einer unterlassenen Information, um daraus ggf. eine Schadensersatzpflicht abzuleiten. Doch will man - von der dogmatischen Begründung abgesehen - überhaupt so weit gehen? Denn wo liegt die Abgrenzung zwischen erwünschten Hinweisen und unerwünschten Einmischungen? Soll die Hinweispflicht mittelbar bedeuten, dass man dazu aufgefordert wird, andere Netzpartner zu denunzieren? Letztlich besteht bei Falschauskünften auch wieder die Gefahr, dass man sich selbst schadensersatzpflichtig macht.

\section{Geheimhaltungspflichten}

Weiter könnten Geheimhaltungspflichten im Netzverbund bestehen. Teubner bringt das Beispiel, dass ein Hersteller know-how oder Modelle eines Zulieferers an einen anderen Lieferanten weitergibt. ${ }^{44}$ Er sieht demgemäß Geheimhaltungspflichten gegenüber Dritten als besondere Verbundpflichten an. ${ }^{45}$ Indes werden sich diesbezügliche Geheimhaltungspflichten schon aus der jeweiligen ausdrücklichen Vertragsvereinbarung ergeben. Fehlen entsprechende Klauseln, kann die Vertragsauslegung u.U. für Geheimnisschutz sprechen. Dabei handelt es sich jedoch nicht um eine netzspezifische Besonderheit; denn solche Geheimhaltungspflichten gegenüber Dritten bzw. Konkurrenten finden sich in Verträgen diverser Art. Außerdem bedarf es zur Feststellung einer solchen Pflicht auch nicht des Rückgriffs auf eine Verbundpflicht oder den Netzzweck.

\section{Gleichbehandlungspflichten}

Außerdem könnte man in Vertragsnetzen an eine Gleichbehandlungspflicht ${ }^{46}$ der Mit- $^{-}$ glieder durch die Systemzentrale denken. Tatsächlich wird die Gleichbehandlung der Systempartner beim Franchising auch dem Interesse der Zentrale entsprechen. Denn damit verbunden ist ein erheblicher Rationalisierungs- und Vereinfachungseffekt. Regelmäßig werden daher bewusst für alle Partner die gleichen Vertragsbedingungen und -vorgaben gewählt. Zugleich wird ein Beitrag zur Einheitlichkeit des Leistungs-

44 Teubner (Fn. 7) 161.

45 Teubner (Fn. 8) 88.

46 Siehe dazu insbesondere Götz Hueck (1958) Der Grundsatz der gleichmäßigen Behandlung im Privatrecht, München: Beck. 
standards im Netz geleistet. Und ferner ist auf diese Weise für Frieden im Netz gesorgt. Doch korrespondiert damit auch eine Gleichbehandlungspflicht? Dagegen spricht zunächst nicht nur der Grundsatz der Vertragsfreiheit, sondern auch der freie Wettbewerb. Anders als im Arbeitsrecht spielen hier weder grundrechtliche Wertungen eine Rolle, noch handelt es sich um unselbstständige abhängige Personen. Die Netzmitglieder sind vielmehr meist rechtlich selbstständige Kaufleute im Sinne der $\S \S 1$ ff. HGB. Demgemäß wird etwa auch im Verhältnis zu Handelsvertretern grundsätzlich nicht von einer Gleichbehandlungspflicht ausgegangen. ${ }^{47}$

Gleichwohl wird geltend gemacht, dass die Gleichbehandlungspflicht hier Ausfluss der Treupflicht sein könne ${ }^{48}$ bzw. dass im Netz gesteigerte Gleichbehandlungspflich$\operatorname{ten}^{49}$ gelten würden. Doch woraus kann sich dies rechtfertigen? Manche verweisen auf den Gleichbehandlungsgrundsatz als Gegenstück zum Weisungsrecht der Zentrale. ${ }^{50}$ In der Tat kann sich insoweit eine Gleichbehandlungspflicht aus der Treupflicht ergeben. Anknüpfungspunkt der Treupflicht ist - bei Arbeitsverhältnissen, aber auch etwa bei Just-in-Time-Verträgen - der Umstand, dass der eine Teil intensiv in die Organisationsstruktur seines Vertragspartners eingebunden und dadurch in besonderer Weise dessen Einfluss und Beherrschung ausgesetzt ist. Korrespondierend zu diesen Einflussmöglichkeiten auf die Interessen und Rechtsgüter des anderen Teils fungiert die Treupflicht als ausgleichendes und begrenzendes Element. Sie fordert Rücksichtnahme auf die integrationsbedingte Abhängigkeit und Unterlegenheit des Vertragspartners und setzt insbesondere willkürlicher Rechtsausübung Grenzen. Die Treupflicht ist Ausdruck einer gesteigerten Pflichtenbindung in solchen langfristigen Vertragsverhältnissen, die in besonderer Weise von kooperativen und integrativen Elementen bzw. von Ausschließlichkeit und Abhängigkeit gekennzeichnet sind. In der Treupflicht verwirklicht sich insoweit die mit spezifischer Rechtsmacht verbundene Verantwortung. Ihre dogmatische Grundlage findet die Treupflicht in $\$ 242$ BGB. ${ }^{51}$ Im neuen deutschen Schuldrecht ist insoweit auch auf § 311 II Nr. 2 BGB zu verweisen, der den Gedanken zum Ausdruck bringt, dass die Einwirkungsmöglichkeit auf Rechte, Rechtsgüter und Interessen eines anderen schutzpflichtbegründend wirken kann.

Wenn die Treupflicht demgemäß willkürlicher Rechtsausübung Grenzen setzt, so verbietet sie auch willkürliche Ungleichbehandlung der Systempartner. Die Treupflicht als Rücksichtnahmepflicht fordert weiter, dass Schaden vom Vertragspartner abgehalten und auf seine Interessen Rücksicht genommen wird. Dem würde es jedoch widersprechen, wenn ein konkurrierender Systempartner von der Zentrale grundlos besser behandelt wird; denn mittelbar ginge dies zu Lasten der anderen Vertragspart-

47 Vgl. BGH BB 1971, 484; Klaus Hopt (1996)»Wettbewerbsfreiheit und Treuepflicht des Unternehmers bei parallelen Vertriebsformen«, 17 ZIP, 1533 m.w.N.

48 Lange (Fn. 22) Rn. 715 für Franchising.

49 Teubner (Fn. 8) 89.

50 Teubner (Fn. 7) 162.

51 Umfassend mit weiteren Nw. Marina Wellenhofer-Klein (1999) Zulieferverträge im Privatund Wirtschaftsrecht, München: Beck, 424 ff., ausführlich zu Treupflichten auch Krebs (Fn. 4) 440 ff., der den Begriff aber noch weiter fasst. 
ner. Positiv formuliert kann sich aus der Treupflicht somit eine Gleichbehandlungspflicht ergeben. So wären etwa den Franchisenehmern gleiche Startbedingungen oder auch gleiche Kündigungskonditionen einzuräumen. Weiterhin wird sich bei Lieferengpässen die Pflicht ergeben, alle Vertragspartner in gleicher Weise anteilig zu beliefern. ${ }^{52}$

Allerdings zeigt sich auch gerade bei der Gleichbehandlungspflicht, dass sie sich ausschließlich im Vertragsverhältnis realisiert und nicht gegenüber sonstigen Netzmitgliedern. Sie setzt zwar begrifflich eine Vielzahl paralleler bzw. sternförmiger Vertragsverhältnisse voraus, die Auswirkungen zeigen sich aber ausschließlich zwischen den jeweiligen Vertragspartnern. Es werden keine Ansprüche zwischen Nichtvertragspartnern begründet. Außerdem führt das Bestehen eines Vertragsverbunds allein auch noch nicht zur Bejahung von Gleichbehandlungspflichten. Denn Voraussetzung ist zwangsläufig das Vorliegen von gleichen Sachverhalten bzw. von Verträgen mit gleichen Leistungsinhalten. Das passt zwar im Bereich des Franchising, aber nicht bei Bauprojekten, wo die beteiligten Unternehmer typischerweise ganz unterschiedliche Leistungen erbringen.

\section{Profit sharing - Vorteilsweiterleitungspflicht}

Mit der sog. Vorteilsweiterleitungspflicht hat sich Teubner im Hinblick auf den vom BGH entschiedenen Apollo-Franchising-Fall beschäftigt. ${ }^{53}$ Hier hatte die Zentrale Mengenrabatte, die sie bei Lieferanten für ihr eigenes Filialsystem durchgesetzt hatte, nicht an die Franchisingpartner weitergegeben. Laut BGH ergab sich die Vorteilsweiterleitungspflicht jedoch aus dem Vertrag und seinen Klauseln. Eben diese Auffassung veranlasste Apollo dann freilich zur umgehenden Änderung der Franchisingverträge. Teubner will demgegenüber die Vorteilsweiterleitungspflicht schon aus dem System heraus ableiten, sodass es auf konkrete Vertragsklauseln gar nicht mehr ankommen würde. ${ }^{54}$ Ein Mengenrabatt sei eben nur erreichbar, wenn eine große Gruppe zusammenwirke. Sei der erreichte Netzvorteil demgemäß dem ganzen Verbund zuzurechnen, so müsse der Vorteil als Netzvorteil aber auch zum Vorteil aller gereichen. ${ }^{55}$ Das Gleichbehandlungsprinzip und Fairnessgesichtspunkte verlangten somit nach einer Aufteilung. ${ }^{56}$

Indes zeigt etwa der Vergleich mit dem Arbeitsrecht, dass Arbeitnehmer keine Erfolgsprämien verlangen können, solange dies nicht konkret vereinbart ist. Rechtfertigen insofern schon die lose Verbundenheit durch den Netzzweck und der Umstand, dass alle Einzelmitglieder auch ein Stück des Risikos mittragen, bereits den Anspruch auf Teilhabe? Oder steht nicht primär dem Franchisegeber als Gründer und Urheber

52 Teubner (Fn. 8) 89.

53 BGH ZIP 2003, 2030 = DB 2003, 2434; ausführlich dazu Reinhard Böhner (2006) »AssetSharing in Franchisenetzwerken - Pflicht zur Weitergabe von Einkaufsvorteilen «, in diesem Heft S. 224 ff., II.4.

54 Teubner (Fn. 8) 80.

55 Teubner (Fn. 8) 96.

56 Teubner (Fn. 7) 169. 
des Systems der Mehrverdienst zu? Meines Erachtens kann sich eine finanzielle Vorteilsweiterleitungspflicht zwar im Einzelfall aus dem einzelnen Vertrag ergeben; doch eine generelle Verbundpflicht dieses Inhalts anzunehmen, überschreitet wohl die Grenzen ergänzender Vertragsauslegung.

\section{Zwischenergebnis}

Es hat sich somit gezeigt, dass die Netzeingebundenheit des einzelnen Vertrags durchaus den Inhalt und die Reichweite der Schutz- und Treupflichten der Vertragspartner beeinflusst. Auch Drittinteressen spielen bei der Inhaltsbestimmung des Pflichtenprogramms mittelbar eine Rolle. Allerdings lässt sich kein fester Katalog von Verbundtreupflichten aufstellen. Dem steht auch die Vielfältigkeit der Netze - z.B. Just-inTime einerseits und Franchising andererseits - entgegen. Im Ergebnis mag man insofern aber mit Teubner ${ }^{57}$ von situativ konkretisierten Verbundtreupflichten sprechen können. Indes bleiben diese Pflichten der Rechtsnatur nach vertragliche Pflichten im Sinne von $§ 241$ II BGB. Bedarf für neue innovative Erklärungsansätze besteht insoweit noch nicht.

\section{Das Rechtsverhältnis von nicht vertraglich miteinander verbundenen Netzmitglie- dern}

\section{Problemkonstellationen}

Es gibt indes Fallkonstellationen, in denen sich die Frage nach unmittelbar drittwirkenden Schutzpflichten und daraus ggf. resultierenden eigenen Schadensersatzansprüchen Dritter mit Nachdruck stellt. Das betrifft einmal das Außenverhältnis, auf das im Weiteren aber nicht näher eingegangen werden soll. ${ }^{58}$ Hier interessiert vielmehr das Binnenverhältnis. So kann man sich im Rahmen eines Franchisingsystems den Fall vorstellen, dass ein Systempartner die vorgegebenen Standards nicht einhält und sich dadurch Kosteneinsparungen und Wettbewerbsvorteile zu Lasten der anderen verschafft. Maßnahmen dieser Art können aber auch dazu führen, dass die Qualität und das Image des Produkts leiden und damit das gesamte System Schaden nimmt. Schreitet hier die Systemzentrale nicht rechtzeitig ein (aus Trägheit, wegen Personalmangels oder aus Respekt vor einem marktmächtigen Partner), so stellt sich die Frage, ob anderen Systempartnern, die Umsatzeinbußen hinnehmen mussten, Ansprüche gegen den Schädiger zustehen. Außerdem sei an den oben ${ }^{59}$ angesprochenen Fall der Kündigung zur Unzeit erinnert. Auch hier geht es um die Frage nach dem Binnendurchgriff im Netz. $^{60}$

57 Teubner (Fn. 8) 86; er geht davon aus, dass diese Verbundtreupflichten im Vergleich zu den anerkannten vertraglichen Treupflichten gesteigert sein sind.

58 Dazu insbesondere Peter Bräutigam (1994) Deliktische Außenhaftung im Franchising, Baden-Baden: Nomos.

59 Siehe oben II.1.b).

60 Dazu Teubner (Fn. 7) 181 ff., 188 ff. 
2. Vertragliche und quasivertragliche Rechtsgrundlagen für eigene Ansprüche dritter Netzmitglieder?

a) Haftung für Erfüllungsgehilfen, § 278 BGB

Keine Aussicht auf Erfolg bietet der Rückgriff auf § 278 BGB, denn der jeweilige Schädiger ist regelmäßig nicht der Erfüllungsgehilfe des eigenen Vertragspartners. Der eine Franchisenehmer ist nicht Erfüllungsgehilfe der Zentrale im Verhältnis zu einem anderen Franchisenehmer. Gleiches gilt im Zulieferbereich. Eine Verschuldenszurechnung auf diesem Wege ist somit ausgeschlossen. ${ }^{61} \mathrm{Im}$ Übrigen würde sie auch gar nicht zu einem Anspruch gegen den Schädiger selbst führen, sondern nur zu einem Anspruch gegen den eigenen Vertragspartner, was regelmäßig nicht gewollt ist.

\section{b) Der Vertrag mit Schutzwirkung zu Gunsten Dritter}

Hoffnungsvoll könnte man weiter an den kraft Richterrechts anerkannten Vertrag mit Schutzwirkung zu Gunsten Dritter denken. ${ }^{62}$ Danach kann sich auch ein Dritter, der nicht Vertragspartner ist, auf den Vertrag bzw. die damit verbundenen Schutzpflichten berufen und daraus Ansprüche ableiten, sofern dieser Dritte mit in den Schutzbereich des Vertrags fällt. Voraussetzungen dafür sind nach h.M. die Leistungsnähe des Dritten, seine Nähe zum Gläubiger, die Erkennbarkeit der Schutzbereichsausdehnung für den Schuldner sowie die Schutzwürdigkeit des Dritten. ${ }^{63}$ Schon letzteres lässt freilich Zweifel aufkommen. Die Schutzwürdigkeit des Dritten könnte bereits deshalb zu verneinen sein, weil ihm ggf. gegen seinen eigenen Vertragspartner inhaltsgleiche Ansprüche zustehen. So dürfte sich nämlich im Franchisingbeispiel durchaus eine Pflicht der Systemzentrale zum Einschreiten gegen den pflichtwidrig handelnden Systempartner bejahen lassen, so dass im Fall des Unterlassens Schadensersatzansprüche denkbar sind.

Die größeren Probleme bereitet jedoch das entscheidende Tatbestandsmerkmal der Leistungsnähe. Es soll den Schuldner vor einer uferlosen Ausdehnung seiner Haftung bewahren. Schutzpflichten sollen nur gegenüber denjenigen Personen bestehen, die bestimmungsgemäß mit der Leistung in Berührung kommen und den Gefahren von Schutzpflichtverletzungen ebenso ausgesetzt sind wie der Gläubiger selbst. ${ }^{64}$ Eine unmittelbare Leistungsberührung findet im Franchisingfall nicht statt. Allerdings zeigt der bekannte Gemüseblattfall ${ }^{65}$, in dem das die Mutter zum Einkauf begleitende Kind auf einem Gemüseblatt ausrutscht und verletzt wird, dass ein unmittelbarer Bezug des Anspruchstellers zur Hauptleistung gar nicht erforderlich ist. Der Geschädigte muss nicht zu den Nutznießern der betreffenden Leistung gehören. Auch muss, wie derselbe Fall zeigt, der Dritte nicht individuell bekannt sein. Allerdings sind die klassischen

61 Vgl. z.B. Hüffer (Fn. 13) 93, 95.

62 Vgl. BGHZ 96, 9, 17, als Grundlage für den Durchgriff der Bankkunden auf andere Banken im Gironetz.

$63 \mathrm{Vgl}$. Heinrichs (Fn. 13) § 328 Rn. 15 ff.

64 BGHZ 49, 354; 70, 329; 129, 168.

65 BGHZ 66, 51 = NJW 1976, 712. 
Schulfälle (Mietverhältnis; Geschäftsanbahnung im Kaufhaus) in spezifischer Weise durch räumliche Nähe geprägt, die in den hier interessierenden Fällen fehlt. Der Bezug des Einzelvertrags zu den anderen Vertragsverhältnissen ist zu lose. Nicht zuletzt aber wird das Rechtsinstitut des Vertrags mit Schutzwirkung auch der Multilateralität von Vertragsnetzen nicht gerecht, da der Blickwinkel immer dem bilateralen Verhältnis verhaftet bleibt. ${ }^{66}$ Ansprüche zwischen Netzmitgliedern, die nicht über Eck miteinander verbunden sind, lassen sich damit eben nicht konstruieren.

\section{c) Sonderverbindung aufgrund Anschlussschuldverhältnisses}

Für eine andere Lösung hat sich Riesenhuber ${ }^{67}$ im Hinblick auf das Rechtsverhältnis bei Nebenparteien ausgesprochen. Er nimmt aufbauend auf der Figur des Vertrags zu Gunsten Dritter eine Sonderverbindung zwischen Nebenparteien an, z.B. zwischen den Mietparteien im selben Mietshaus oder den Arbeitnehmern desselben Betriebs. Hier könne sich, wenn die Anlage des Anschlussschuldverhältnisses die Einbeziehung des Dritten rechtfertige, für diesen eine Schutzwirkung ergeben. Der Schutz des Dritten sei als Teilhabe an dem Anschlussschuldverhältnis zu qualifizieren und daraus auch zu entwickeln. ${ }^{68}$ Die Kriterien Gläubiger- und Leistungsnähe seien dafür Indizien, aber nicht zwangsläufige Voraussetzungen. Der für die Schutzwirkung entscheidende Umstand scheint vielmehr die vertraglich bestimmte Einordnung in eine Gemeinschaft ${ }^{69} \mathrm{zu}$ sein. ${ }^{70}$ Das Zusammentreffen und -wirken der Personen muss bestimmungsgemäß sein und nicht nur zufällig. ${ }^{71}$ Dadurch werde der Rechtskreis des Einzelnen in einen einheitlichen Rechtskreis der Gemeinschaft integriert und infolgedessen von der Gemeinschaft mitbestimmt. ${ }^{72}$ Die Reichweite der Pflichten beurteile sich dann nach vertraglicher Vereinbarung, z.B. je nach Art der Tätigkeit des Einzelnen (z.B. Arbeitnehmers) und seinem Einwirkungsbereich. ${ }^{73}$ Denkbar sind Rücksichtnahmepflichten, Geheimhaltungspflichten oder auch Verkehrssicherungspflichten.

Die Überlegungen Riesenhubers leuchten durchaus ein. Allerdings orientiert sich sein Modell in erster Linie an Mietnachbarn und Arbeitnehmern. Diese Rechtsverhältnisse sind durch tatsächliche räumliche Nähe gekennzeichnet. Und eben daraus resultieren Berührungsflächen und gegenseitige Einwirkungsmöglichkeiten, die wiederum nach korrespondierenden Schutzpflichten verlangen. ${ }^{74}$ Das passt im Vertragsverbund aber nur begrenzt. Das Bild mag passen im Fall größerer Bauprojekte, wo viele Unternehmer bzw. Handwerker nebeneinander auf derselben Baustelle tätig sind. Anders liegt es jedoch etwa im Bereich des Franchising. Hier geht es nur um ein mittelbares

66 Zutreffend Teubner (Fn. 7) $230 \mathrm{f}$.

67 Karl Riesenhuber (1997) Die Rechtsbeziehungen zwischen Nebenparteien, Berlin: Duncker \& Humblot.

68 Riesenhuber (Fn. 67) 166.

69 Womit keine Rechtsgemeinschaft im technischen Sinne gemeint ist, vgl. S. 204.

70 Riesenhuber (Fn. 67) 181.

71 Riesenhuber (Fn. 67) 188.

72 Riesenhuber (Fn. 67) 205.

73 Riesenhuber (Fn. 67) 182.

74 Riesenhuber (Fn. 67) 207. 
und typischerweise auch nur um ein wirtschaftliches Betroffensein anderer Netzmitglieder. Ob dies ausreichend sein kann, fragt sich auch im Hinblick darauf, dass die Franchisenehmer zugleich regelmäßig miteinander in Wettbewerb stehen. Die Bestimmung des Pflichtenprogramms fällt daher ungleich schwerer als zwischen den von Riesenhuber untersuchten Nebenparteien. Und nicht zuletzt ist zu beachten, dass der Ansatz von Riesenhuber wiederum bilateral bzw. trilateral angelegt ist. Wie schon zuvor beim Vertrag mit Schutzwirkung für Dritte lassen sich damit Ansprüche zwischen Netzmitgliedern, die nicht über Eck miteinander verbunden sind, nicht begründen.

\section{d) Schutzpflichtverhältnis gemäß $§ 311$ II Nr. 3 BGB}

Verlockend ist der Gedanke, im Verhältnis der nicht vertraglich miteinander verbundenen Netzmitglieder ein Schutzpflichtverhältnis im Sinne von § 311 II Nr. 3 BGB anzunehmen. Danach kann ein Schuldverhältnis mit Pflichten nach § 241 II BGB, also mit Schutzpflichten, auch durch »ähnliche geschäftliche Kontakte« entstehen. Es wird also gerade kein Vertrag vorausgesetzt, sondern nur ein ähnlicher, vertrauensbegründender Kontakt. Um einen Kontakt, der auf den Abschluss eines Vertrags abzielt, muss es gerade nicht gehen. ${ }^{75}$ Ausreichend ist zum Beispiel der Kontakt im Rahmen eines Gefälligkeitsverhältnisses. ${ }^{76}$ Das Tatbestandsmerkmal »geschäftlich« in Abgrenzung zu rein sozialen Kontakten macht vorliegend wenig Schwierigkeiten. Problematisch ist jedoch das Erfordernis des Kontakts selbst. Kontakt steht dem Wortlaut nach für unmittelbare Berührung und Begegnung. Daran fehlt es, wenn sich Pflichtverletzungen erst im Rahmen einer Kettenreaktion oder nur reflexartig bei einem Dritten auswirken, so wie im obigen Fallbeispiel. Außerdem erfordert die Formulierung »geschäftlicher Kontakt« eine mehr oder weniger zielgerichtete, bewusste Begegnung. Daher bleibt nur das Fazit, dass der Gesetzgeber hier zwar die Möglichkeit hätte, die Verbundenheit im Netz als weiteren Schutzpflichttatbestand in $§ 311$ BGB aufzunehmen. Mit dem geltenden Wortlaut lässt sich die Problematik im Netz jedoch noch nicht erfassen.

e) Die Forderung Teubners nach Annahme einer Sonderverbindung im Vertragsverbund Auch Teubner sucht die Lösung über die Annahme einer Sonderverbindung. ${ }^{77}$ Seiner Meinung nach begründet der Vertragsverbund eine Pflichten begründende Sonderverbindung ähnlich der culpa in contrahendo (cic). ${ }^{78}$ Die Rechtfertigung dafür sieht er in folgenden drei Aspekten, die zugleich als wesentliche Kennzeichen des Vertragsverbunds begriffen werden: (1) die wechselseitige Verweisung der bilateralen Verträge aufeinander im Leistungsprogramm; (2) der inhaltliche Bezug der Verträge auf das gemeinsame Projekt (Verbundzweck); und (3) die enge Kooperationsbeziehung zwischen den Verbundbeteiligten. ${ }^{79}$

75 Heinrichs (Fn. 13) § 311 Nr. 18.

76 Heinrichs (Fn. 13) § 311 Nr. 18 und Einl. 8 vor $§ 241$.

77 Umfassend zur Lehre von den Sonderverbindungen: Krebs (Fn. 4).

78 Teubner (Fn. 7) $201 \mathrm{f}$.

79 Teubner (Fn. 7) 125. 
Hierauf aufbauend will Teubner dem systemtreuen Franchisenehmer in Anlehnung an die gesellschaftsrechtlichen Grundsätze über die actio pro socio die Geltendmachung der Ansprüche des Franchisegebers gegen den »Freerider « auf Einhaltung der Systemstandards im eigenen Namen erlauben, wenn der Franchisegeber aufgrund opportunistischen Verhaltens oder kollusiven Zusammenwirkens mit dem »Freerider « untätig bleibt. Dabei geht es indes nur um die Geltendmachung von Ansprüchen, deren Erfüllung dem Erreichen des gemeinsamen Netzzwecks dient. ${ }^{80}$ Die Geltendmachung eines individuellen Schadens ist über die Rechtsfigur der actio pro socio nicht möglich. Die Aktion kann allerdings vorbeugend zur Schadensvermeidung dienen.

\section{Die Anwendung von $\S 826 B G B$}

a) Einführung

Im Hinblick auf den Binnendurchgriff hat sich das Vertragsrecht somit als wenig ergiebig erwiesen. Hier führen weder die vertraglichen noch die anerkannten quasivertraglichen Anspruchsgrundlagen zur Bejahung von Schadensersatzpflichten. Somit bleibt die Frage, ob und inwieweit im Einzelfall deliktsrechtliche Ansprüche in Betracht kommen mögen. Da es meist nur um Vermögensbeschädigungen geht, scheidet $\S 823$ I BGB als Anspruchsgrundlage regelmäßig aus. Zumindest ein Teil der als kritisch erachteten Fälle könnte jedoch in den Anwendungsbereich von $§ 826$ BGB fallen. Danach ist zum Schadensersatz verpflichtet, wer in einer gegen die guten Sitten verstoßenden Weise einem anderen vorsätzlich Schaden zufügt. Es genügt ein reiner Vermögensschaden. Ob der Schaden unmittelbar oder nur mittelbar auf der Handlung des Schädigers beruht, ist irrelevant. Somit kommen auch die zuvor genannten Fallkonstellationen wie Schadenszufügung infolge einer Kündigung zur Unzeit oder durch fortgesetzte Verstöße gegen die Vorgaben des Franchisesystems grundsätzlich für $\S 826$ BGB in Betracht.

\section{b) Der Verstoß gegen die guten Sitten}

Zweite Voraussetzung von $\S 826$ BGB ist, dass die Schadenszufügung durch eine sittenwidrige Handlung bewirkt wurde. Sittenwidrigkeit wird allgemein als Verstoß gegen das Anstandsgefühl aller billig und gerecht Denkenden definiert. ${ }^{81}$ Entscheidend ist dabei der betroffene Verkehrskreis. ${ }^{82}$ Es ist auf die in der beteiligten Gruppe anerkannten moralischen Anschauungen abzustellen. ${ }^{83}$ Im Unternehmensbereich etwa spielen demgemäß auch die Handelsbräuche und konkreten Verkehrssitten der Branche eine wichtige Rolle.

Daran knüpft sich im vorliegenden Zusammenhang die Frage nach den Verkehrssitten und Verhaltenserwartungen im Netz. Diese richten sich zwar im Einzelfall nach der Art des konkreten Netzes und der jeweiligen Branche. Indes lässt sich, aufbauend auf den

80 Teubner (Fn. 7) $209 \mathrm{f}$.

81 RGZ 80, 221; BGHZ 10, 228, 232; 69, 297; BGH NJW 2004, 2668, 2670.

82 BGHZ 10, 228, 232.

83 Heinrichs (Fn. 13) § 138 Rn. 2 
bisherigen Untersuchungen zum Recht der Vertragsnetzwerke, doch ein Grundbestand, ein Mindeststandard an Verhaltenserwartungen und -notwendigkeiten formulieren, der allgemein für die Funktionsfähigkeit von Vertragsverbünden vorausgesetzt wird und somit Ausdruck von Verkehrssitten im Netz ist. Ausgangspunkt ist dabei, dass die mit dem Vertragsverbund verbundenen Vorteile (Mengeneffekte; Kostensenkung; Risikoverteilung; Flexibilität u.a.) nur erreichbar sind, wenn alle in fairer Weise mitwirken. Kennzeichnend ist insofern der Umstand, dass sich alle Netzmitglieder mit dem Eintritt in den Verbund zwangsläufig dem Einfluss der anderen Mitglieder aussetzen und somit, wenn auch in Gewinnerwartung, die Risiken des Netzverbundes bewusst in Kauf nehmen. Manche hängen sogar investitionsbedingt existentiell von der Funktionsfähigkeit des Systems ab. Demgemäß bietet das Netz durchaus auch »Freiräume für verantwortungslose Interaktionen $\ll .{ }^{84}$ Die Risiken werden aber akzeptiert, weil allseits - soweit der Netzzweck reicht - die Erwartung von Fairness besteht. Es wird implizit erwartet, dass man sich an die jeweiligen Spielregeln des Systems hält und sich nicht rücksichtslos auf Kosten der anderen oder auf Kosten des Gesamtsystems Vorteile verschafft.

Hier passt der Vergleich mit einem sportlichen Wettkampf. Unabhängig davon, ob man in der gleichen Mannschaft oder in konkurrierenden Mannschaften steht, und unabhängig davon, welche individuellen Interessen verfolgt werden, vertrauen doch alle Spieler darauf, dass die grundlegenden Regeln von allen eingehalten werden. Sonst wäre, zumal keine vertragliche Verbindung besteht, niemand bereit, mitzumachen und eigene Rechtsgüter - wie im Sport Körper und Gesundheit - aufs Spiel zu setzen. Und zu den Regeln und Erwartungen gehört insoweit auch, dass zwar nicht leichtere Unregelmäßigkeiten, wohl aber klare Pflichtverstöße (»Fouls«) entsprechend geahndet werden.

Auf das Netz bezogen heißt das, dass wegen system- und wettbewerbsimmanenter »üblicher« Gefährdungen und Schäden keine Schadensersatzansprüche geltend gemacht werden könnten, wohl aber in Fällen, in denen eine »erhebliche Gefährdung der Grundlage des Gesamtsystems ${ }^{85}$ in Kauf genommen wird. Dann läge auch ein Verstoß gegen das Anstandsgefühl aller billig und gerecht denkenden Netzmitglieder vor. Ein grundlegender rechtswidriger »Spielregelverstoß« könnte daher als Sittenwidrigkeit im Sinne von $\$ 826$ BGB zu werten sein.

\section{c) Der subjektive Tatbestand}

Die dritte und letzte Voraussetzung von $§ 826$ BGB betrifft den Vorsatz. Der eingetretene Schaden muss vom Vorsatz des Schädigers mit umfasst sein. Der Täter muss Kenntnis von dem Eintritt eines Schadens, von der Kausalität des eigenen Verhaltens und den die Sittenwidrigkeit des Verhaltens begründenden Tatsachen haben. ${ }^{86}$ Es ist allerdings nicht erforderlich, dass gerade die Absicht bestand, den anderen zu schädi-

84 Eduard Picker (1999) »Gutachterhaftung, Außervertragliche Einstandspflichten als innergesetzliche Rechtsfortbildung «, in: Volker Beuthien (Hg.) Festschrift für Dieter Medicus, Köln: Heymanns, 397-447, 432, im Hinblick auf Gutachterhaftung.

85 Vgl. BGH NJW 1999, 1177, 1178.

86 Wagner (2004) »§ 826«, in: Franz Säcker und Kurt Rebmann (Hg.) Münchener Kommentar zum Bürgerlichen Gesetzbuch, 4. Aufl., München: Beck, § 826 Rn. 20. 
gen. Insoweit genügt bedingter Vorsatz. ${ }^{87}$ Auch muss die geschädigte Person nicht individuell bekannt sein. ${ }^{88}$ Der subjektive Tatbestand wirft insoweit kaum Probleme auf und dürfte in den hier interessierenden Fallkonstellationen regelmäßig erfüllt sein.

\section{d) Abschließende Würdigung}

Folglich kann $\S 826$ BGB als Grundlage für Schadensersatzansprüche im Netz bzw. für den Binnendurchgriff grundsätzlich in Betracht kommen. Es ist allerdings Vorsicht geboten. § 826 BGB kann nicht als Allzweckwaffe dienen. Es handelt sich um eine Ausnahmevorschrift, die den Grundsatz von $\S 823$ BGB, dass allein spezifische subjektive Rechtsgüter dem deliktischen Schutz unterliegen, nur für den eher seltenen Fall einer vorsätzlichen sittenwidrigen Schädigung durchbricht. Nur unter diesen engen Voraussetzungen soll es zu einer Haftungsausdehnung auf Vermögensschäden kommen; denn alles andere würde zu einer unerträglichen und unberechenbaren Einschränkung der Handlungsfreiheit bzw. unternehmerischen Entscheidungsfreiheit führen. ${ }^{89}$

Demnach darf die Anwendung von § 826 BGB insbesondere nicht bewirken, dass eine etwaige vertragliche Risikoverteilung unterlaufen wird. Weiterhin dürfen zwangsläufige Konsequenzen von fairem Wettbewerbsverhalten nicht erfasst werden. ${ }^{90}$ Eine wettbewerbskonforme »Schädigung « von Konkurrenten ist nämlich durchaus erwünscht, da gesamtwirtschaftlich von Vorteil. Ferner darf die Anwendung von $\S 826$ BGB nicht dazu führen, dass diffuse Schäden erfasst werden, die in einer schwer messbaren Form bei einer Vielzahl von Personen eintreten. ${ }^{91}$ Und nicht zuletzt muss einer beliebigen Haftungsausdehnung auf nur mittelbar geschädigte Personen entgegengewirkt werden.

Indes scheinen diese Grenzen im Fall einer Haftung gegenüber Netzmitgliedern grundsätzlich nicht überschritten zu sein. Der Kreis der potentiell Betroffenen und möglichen Schadensersatzgläubiger ist von vornherein fixiert und auch überschaubar. ${ }^{92}$ Eine übermäßige Haftungsausdehnung wird dadurch vermieden, dass nur besonders schwerwiegende Pflichtverletzungen erfasst werden können, die die Gefahr einer erheblichen Beeinträchtigung des Netzzwecks und daraus resultierender Schäden begründen. ${ }^{93}$ Hinzu kommt, dass der Geschädigte die volle Darlegungs- und Beweislast für alle Anspruchsvoraussetzungen von $\S 826 \mathrm{BGB}$ trägt ${ }^{94}$, so dass mit mutwilligen Klagen von mittelbar und nur geringfügig Geschädigten ohnehin nicht zu rechnen ist.

Die Anwendung von $\S 826$ BGB hat insoweit auch den Vorteil, dass sie dogmatisch fragwürdige Schutzbereichserweiterungen ${ }^{95}$ und auch die Schaffung neuer Sonderver-

87 BGH NJW 2000, 2896; 2004, 446 und 2664.

88 BGH VersR 1956, 641.

89 Vgl. auch Picker (Fn. 84) 437.

90 Wagner (Fn. 86) § 826 Rn. 12 f.

91 Wagner (Fn. 86) § 826 Rn. 16.

92 Vgl. zu diesem Erfordernis im Hinblick auf die Gefahr einer übermäßigen Haftungsausdehnung auch Picker (Fn. 84) 437 f. zur Gutachterhaftung.

93 Vgl. Teubner (Fn. 7) 207 für vertragslose Verbundpflichten.

94 BGH NJW 2004, 2664, 2665.

95 Dazu ausführlich das Werk von Krebs (Fn. 4). 
bindungen vermeidet. ${ }^{96}$ Zudem zeigt ein Blick in die Rechtsprechung, dass § 826 BGB gerade in Fällen herangezogen wird, die sich durch Drei- und Mehrpersonenverhältnisse auszeichnen. So hat die Rechtsprechung die Haftung von Wirtschaftsprüfern und Steuerberatern lange Zeit mit Hilfe von $\S 826$ BGB begründet. ${ }^{97}$ Erst in jüngerer Zeit wurde dann auch auf den Vertrag mit Schutzwirkung für Dritte zurückgegriffen. ${ }^{98}$ Ähnliches gilt für Fälle der Haftung von Wertgutachtern und Sachverständigen, die ebenfalls an der Schnittstelle von Schutzwirkungskonstruktionen und § 826 BGB liegen. ${ }^{99}$ Und nicht zuletzt werden im Gesellschaftsrecht Maßnahmen von Einzelgesellschaftern zu Lasten der Gesellschaft außer mit der Konstruktion über die Verletzung der gesellschaftsrechtlichen Treupflicht auch mit $\S 826$ BGB zu erfassen versucht. ${ }^{100}$ Das gilt etwa für den Fall, dass ein Geschäft statt im Namen der Gesellschaft in eigenem Namen abgewickelt wird. ${ }^{101} \S 826$ BGB ist daher - in seinen freilich engen Schranken - in besonderer Weise auch dazu geeignet, rücksichtslose Schädigungen an Dritten zu erfassen, die außerhalb von Vertragsverhältnissen stehen.

\section{Fazit}

Der Umstand, dass die Einbindung eines Vertrags in einen Gesamtverbund von Verträgen Auswirkungen auf die Interpretation und Inhaltsbestimmung der beiderseitigen Rechte und Pflichten hat, wurde hier nicht mehr in Frage gestellt, sondern vielmehr schon vorausgesetzt. Insoweit zeigt sich, dass der Netzkontext die vertraglichen Schutzpflichten in spezifischer Weise ausformt. Die »Drittwirkung « prägt die vertraglichen Pflichten mit, die Auswirkungen bleiben aber auf das bipolare Vertragsverhältnis beschränkt. Es werden keine unmittelbaren Rechte oder Pflichten von Dritten auf Basis oder im Zusammenhang mit dem einzelnen Vertrag begründet. Dafür besteht auch - soweit ersichtlich - kein Bedürnnis.

Vertrags- bzw. pflichtwidriges Verhalten, das bei anderen Mitgliedern des Vertragsverbunds, also bei Nicht-Vertragsbeteiligten, zu Schäden führt, kann aber im Einzelfall die Voraussetzungen von $\$ 826$ BGB erfüllen und so zu einer Schadensersatzpflicht des Schädigers führen. Die engen Tatbestandsvoraussetzungen des § 826 BGB übernehmen dabei die notwendige Selektionsfunktion. Es werden nur die »harten« Fälle erfasst, in denen Schädiger, Geschädigter und Schaden klar zu erfassen sind und die Schwere des Pflichtverstoßes auf der Hand liegt. Es sind dann zugleich die Fälle, in denen es auch angemessen erscheint, auf das jeweilige Problem tatsächlich mit rechtlichen Mittel ${ }^{102}$ zu reagieren. Was dabei im Einzelnen als Mindeststandard von Verhaltenserwartungen und -notwendigkeiten formuliert werden kann und somit als

96 Dagegen auch Bräutigam (Fn. 58) 40, im Hinblick auf Franchising.

97 BGH NJW 1973, 321; 1987, 1758; 1992, 2080; OLG München WM 1997, 613, 620.

98 BGHZ 138, 257, 260 f.= NJW 1998, 1948.

99 BGH NJW 1991, 3282, 3283.

100 BGH NJW 1992, 2821. Näher Wagner (Fn. 86) § 826 Rn. 94 ff. m.w.N.

101 RGZ 89, 99, 103 f.

102 Zur Frage, ob außerrechtliche Sanktionsmechanismen in Vertragsnetzen genügen, auch Collins (Fn. 1). 
Ausdruck der Verkehrssitten im Netz zu begreifen ist, bedarf allerdings noch weiterer Konkretisierung. Dieser Prozess kann angesichts der Vielgestaltigkeit von Vertragsnetzen auch nur gruppenbezogen erfolgen. Der Fall, dass ein Netzmitglied durch eine plötzliche Kündigung ${ }^{103}$ oder eine Kündigung zur Unzeit bewusst die Schädigung anderer Netzmitglieder in Kauf nimmt, oder auch der Fall, dass ein »Freerider « sich absichtlich auf Kosten der anderen Unternehmer Vorteile verschafft, sind aber grundsätzlich geeignet, den Tatbestand von $§ 826$ BGB zu erfüllen.

103 Siehe den »Kühltransportefall« BGH NJW 1999, 1177. 